ARTICLE

\title{
The tomato receptor CuRe1 senses a cell wall protein to identify Cuscuta as a pathogen
}

Volker Hegenauer (10 1,2, Peter Slaby ${ }^{1}$, Max Körner ${ }^{1}$, Julien-Alexander Bruckmüller (1) 3,9, Ronja Burggraf ${ }^{2}$, Isabell Albert ${ }^{1}$, Bettina Kaiser ${ }^{2}$, Birgit Löffelhardt ${ }^{2}$, Irina Droste-Borel ${ }^{4}$, Jan Sklenar (1) ${ }^{5}$, Frank L. H. Menke (D) ${ }^{5}$, Boris Maček (10 4 , Aashish Ranjan (1) 6,7, Neelima Sinha (1) 6, Thorsten Nürnberger ${ }^{2,8}$, Georg Felix²,

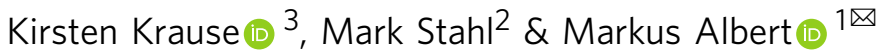

Parasitic plants of the genus Cuscuta penetrate shoots of host plants with haustoria and build a connection to the host vasculature to exhaust water, solutes and carbohydrates. Such infections usually stay unrecognized by the host and lead to harmful host plant damage. Here, we show a molecular mechanism of how plants can sense parasitic Cuscuta. We isolated an $11 \mathrm{kDa}$ protein of the parasite cell wall and identified it as a glycine-rich protein (GRP). This GRP, as well as its minimal peptide epitope Crip21, serve as a pathogen-associated molecular pattern and specifically bind and activate a membrane-bound immune receptor of tomato, the Cuscuta Receptor 1 (CuRe1), leading to defense responses in resistant hosts. These findings provide the initial steps to understand the resistance mechanisms against parasitic plants and further offer great potential for protecting crops by engineering resistance against parasitic plants.

\footnotetext{
${ }^{1}$ Department of Biology, Molecular Plant Physiology, Staudtstraße 5, 91058 Erlangen, Germany. ${ }^{2}$ Center for Plant Molecular Biology, Auf der Morgenstelle 32, 72076 Tübingen, Germany. ${ }^{3}$ Department of Arctic and Marine Biology, Breivika, 9037 Tromsø, Norway. ${ }^{4}$ Quantitative Proteomics \& Proteome Center Tübingen, Auf der Morgenstelle 15, 72076 Tübingen, Germany. ${ }^{5}$ The Sainsbury Laboratory, University of East Anglia, Colney Lane, NR4 7 UH Norwich, UK. ${ }^{6}$ Department of Plant Biology, College of Biological Sciences, UC Davis, Davis, CA, USA. ${ }^{7}$ National Institute of Plant Genome Research, New Delhi, India. ${ }^{8}$ Department of Biochemistry, University of Johannesburg, Johannesburg 2001, South Africa. ${ }^{9}$ Present address: Solana Research GmbH, Eichenallee 9 , D 24340 Windeby, Germany. ${ }_{\text {email: markus.albert@fau.de }}$
} 
C haracteristic molecular patterns uncover pathogens as external invaders and are critical signatures that are detected by the innate immune system of both, animals and plants. This discrimination between self and non-self allows the host organisms to initiate defense reactions and resist pathogen attacks. As part of their innate immune system, plants evolved cell surface receptors to detect molecular patterns ${ }^{1-3}$. Due to the facts that most plant pathogens are microbes or arthropods and the wider evolutionary distance between plants and those attackers, the presence of "plant pattern recognition receptors" to detect molecular patterns seems a logical consequence of evolution. However, $\sim 4500$ higher plant species live as parasites and thus pose an additional threat to plants. Well-known parasitic plants with high agronomical relevance are Striga spp., Orobanche spp., and Cuscuta spp $^{4-6}$. Most host plants are unable to detect an invasion by parasitic plants and the attackers stay unrecognized due to the limited innate immune system of host plants for detecting dangerous parasitic plants. Yet, a few host exceptions are described, which are able to fend off parasitic plants and stay incompatible ${ }^{7-9}$. However, the molecular mechanisms behind these are poorly understood, and molecular patterns of parasitic plants, which could mark a plant parasite as a devastating invader, have not yet been described.

Cuscuta spp. are holoparasitic plants which infect a broad spectrum of hosts by connecting to their vasculature via specific feeding structures, called haustoria (Fig. 1a, b) ${ }^{10-12}$. Cultivated tomato (Solanum lycopersicum) is one of few host exceptions that recognizes Cuscuta reflexa as an alien invader and actively initiates defense responses ${ }^{4,13,14}$ measureable as the emission of the stress-phytohormone ethylene or reactive oxygen species (ROS) in tomato leaves ${ }^{15}$ and visible as hypersensitive response (HR) at the infection sites (Fig. 1c) ${ }^{13-15}$. We reported the tomato cell surface receptor "Cuscuta receptor 1" (CuRe1) as a critical component for the detection of C. reflexa due to a hypothesized Cuscuta factor or pathogen-associated molecular pattern (PAMP) that can be extracted from the parasitic plant and triggers the defense response in a CuRe1-dependent manner. This Cuscuta factor is a heat stable protein and sensitive to treatments with bases $(\mathrm{pH}>11)$, indicating potential secondary modifications present on the peptide backbone ${ }^{16}$. The Cuscuta factor is found in all organs of C. reflexa irrespective of its infectious stage and seems to locate to the parasite's cell wall ${ }^{15}$. Here, we purified this Cuscuta factor from $C$. reflexa extracts and identified it as a Glycine-rich protein (GRP). We further characterized its function as a binding ligand for CuRe1 and the specifically triggered plant defense responses.

\section{Results and discussion}

Purification and identification of a defense-triggering Cuscuta factor. Since we knew that the Cuscuta factor originates from the cell wall, we focused on extracts prepared from the parasite cell wall and tested them for bioactivity in the ethylene bioassay specifically induced via CuRe1 (Fig. 1d). Compared to incubation in buffer/water alone, higher amounts of Cuscuta factor were found to be released from cell wall fractions by treatments with pectinases and, with much lower efficiency, by cellulases (Fig. 1d). Both types of enzymes are known to be present and active in penetrating Cuscuta spp. haustoria and can thus lead to an increased release of the Cuscuta factor from the cell walls of intruding haustoria during the infection process ${ }^{17,18}$.

To extract sufficient amounts of the Cuscuta factor from collected plant material, we scaled up the previous protocol ${ }^{15}$ and used acidic extraction conditions $(0.1 \mathrm{M} \mathrm{HCl}, \mathrm{pH} 1)$. The analysis was also extended to all of the activities that eluted as distinct peaks from the first cation exchange column (Supplementary Fig. 1).
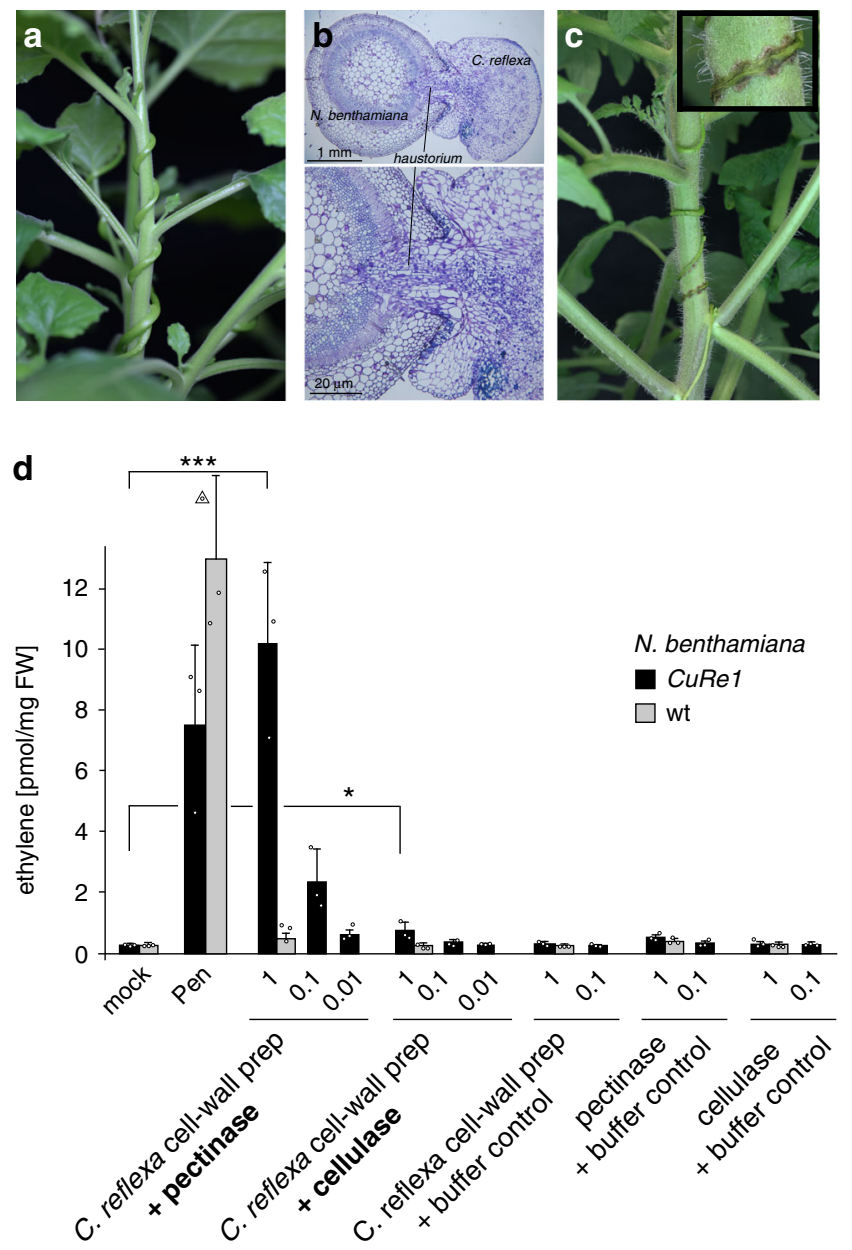

Fig. 1 Cuscuta reflexa induces defense in cultivated tomato by a pathogen-associated molecular pattern. a $C$. reflexa can infect nearly all dicot plants as susceptible hosts; picture shows infected Nicotiana benthamiana; b Parasitic haustoria successfully penetrate the $N$. benthamiana shoot; c $\mathrm{C}$. reflexa induces visible hypersensitive response (HR) on tomato (S. lycopersicum) shoot at the contact sites of the parasite's haustoria. d C. reflexa cell wall preparations were treated with either cellulase or pectinase; extracts were applied to trigger defense-related ethylene biosynthesis in transgenic CuRe1-expressing N. benthamiana plants. Numbers on $x$-axis indicate applied extract volume in $\mu \mathrm{l}$; bovine serum albumin (BSA; $0.01 \mathrm{mg} / \mathrm{ml}$ ) buffered in $25 \mathrm{mM} \mathrm{MES} \mathrm{(} \mathrm{pH}$ 5.7) was added as mock control; Penicillium extract $(0.05 \mathrm{mg} / \mathrm{ml})$ served as positive control ${ }^{31}$. $\mathrm{FW}$, fresh weight. Ethylene measurements show means of three technical replicates; error bars denote SD. Wildtype (wt) N. benthamiana plant samples have not been tested with diluted extract preparations $(0.1 \mu \mathrm{l}$ and $0.01 \mu \mathrm{l})$ since they did not respond to maximum doses $(1 \mu \mathrm{l})$ in the ethylene assay. Dots indicate single data points, triangle shows outlier at 16.87; Asterisks show Student's $t$ test; ${ }^{\star \star \star} p<0.0028$; ${ }^{\star} p<0.0285$; representative graphs are shown; all experiments were repeated more than three times.

When purifying the extracts by cation exchange or reversed phase chromatography, the Cuscuta factor activity detectable by the CuRe1 receptor eluted in several peaks, indicating presence of activity in structurally different forms (Supplementary Fig. 1) ${ }^{15}$. We further purified and enriched the Cuscuta factor(s) from the obtained fractions and performed LC-MS/MS analyses for each sample individually. Several distinct masses correlated with CuRe1dependent bioactivity and we identified 11 different compounds all of which represented active forms of the Cuscuta factor (Supplementary Fig. 2). MS/MS fragmentation studies of the correlated candidate masses shared similar fragment peaks 
a C. reflexa Glycine-rich Protein (CrGRP), aa-sequence

targeting sequence

\begin{tabular}{ccccccc}
1 & 10 & 20 & 30 & 40 & 50 & 60 \\
1 & 1 & 1 & 1 & 1 & 1 & 1 \\
\hline & MSSRVFLLLGLSLALSLMIASEVTRARELAETTTAASFDTNGYGHGKHGGSHGWHGGHGGG
\end{tabular}

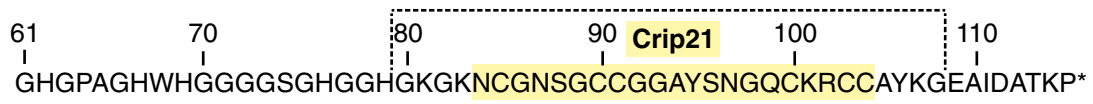

sequenced by MS/MS inconclusive fragmentation

b

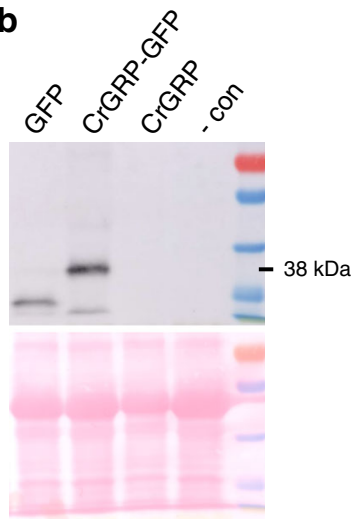

C

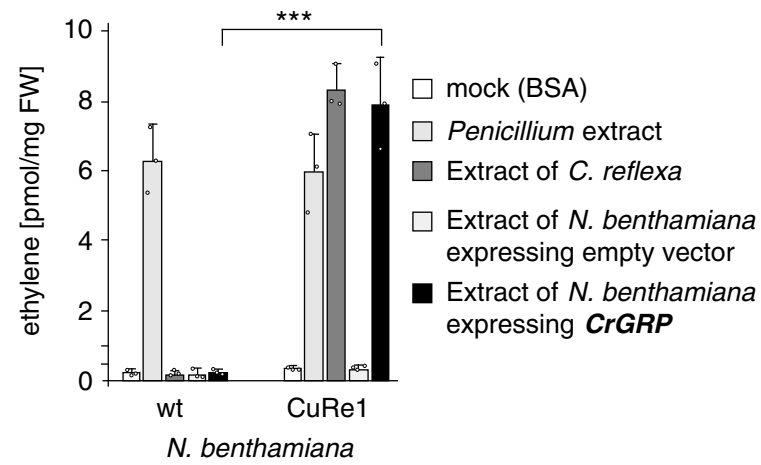

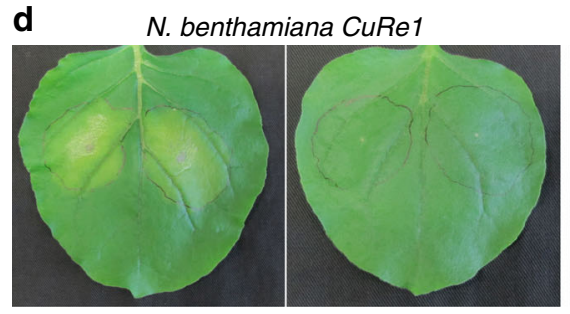

+ CrGRP-GFP

+ GFP

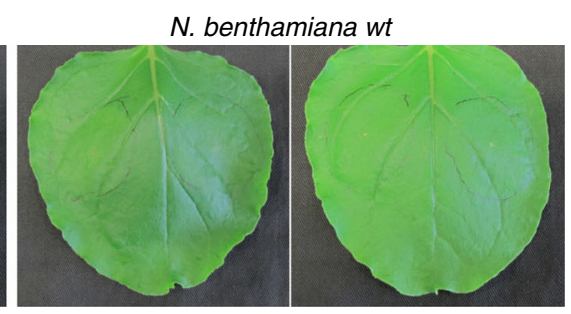

+ CrGRP-GFP $\quad+$ GFP

Fig. 2 The C. reflexa Glycine-rich protein (CrGRP) triggers CuRe1-dependent defense responses. a Protein sequence of the identified CrGRP; the peptide isolated from C. reflexa extracts was sequenced de novo by mass spectrometry and is indicated starting at aa-position 70, dashed line indicates the peptide part which could not be sequenced by MS/MS; Crip21 motif highlighted in yellow. b heterologous expression of CrGRP gene in N. benthamiana leaves; WB shows a c-terminally GFP-tagged CrGRP. c Ethylene response in leaves of wt or CuRe1-expressing $\mathrm{N}$. benthamiana. Plants were treated with extracted CrGRP after heterologous expression shown in b. Bovine serum albumine (BSA; $0.01 \mathrm{mg} / \mathrm{ml}$ ) buffered in $25 \mathrm{mM}$ MES ( $\mathrm{pH}$ 5.7) was added as mock control; Penicillium extract $(0.05 \mathrm{mg} / \mathrm{ml})$ served as positive control ${ }^{31}$. FW, fresh weight; ethylene measurements show means of three technical replicates; dots indicate single data points; error bars denote SD, asterisks denote student's t-test, $p \leq 0.0003$; representative graphs are shown; experiments were repeated more than three times. $\mathbf{d}$ Expression of CrGRP-GFP in leaves of wildtype (wt) and transgenic CuRe1-expressing N. benthamiana plants. GFP alone served as negative control; pictures 5 days after expression.

(Supplementary Fig. 2) and had the fragment mass of $2077 \mathrm{Da}$ in common. Another characteristic feature of all corresponding MSspectra was an inconclusive fragmentation pattern with only a few clear but characteristic fragment masses (Supplementary Fig. 2) that have been previously observed ${ }^{15}$. Since the commonly present fragment of $2077 \mathrm{Da}$ is a y-fragment and the spectra of the heavier candidate masses contained the lighter candidate masses as their $y$ fragments, we assumed a common origin of all fragments from the same protein. The mass differences of the N-terminal fragmentations could be correlated to those of single amino acid residues, which allowed us to deduce the N-terminal part of the peptide sequence (Supplementary Fig. 2). The information obtained from overlays of seven individual MS/MS fragmentation analyses in four individual LC-MS/MS runs revealed the sequence of the first $15 \mathrm{~N}$ terminal amino acids of the peptide (Fig. 2a and Supplementary Fig. 2). A p-blast search against a translated transcriptome database from C. reflexa ${ }^{19}$ resulted in a perfect hit on a glycine-rich protein (GRP) of C. reflexa (Fig. 2a). CrGRP consists of 116 amino acids with an n-terminal targeting sequence that predicts an extracellular localization (Fig. 2a). According to the current classification of GRPs $^{20}$, the CrGRP belongs to the class II which comprises a distinguishing c-terminal cysteine-rich region (Fig. $2 \mathrm{a}$ ). We cloned the corresponding CrGRP gene from $C$. reflexa genomic DNA and transiently expressed it in $N$. benthamiana leaves for $\sim 72 \mathrm{~h}$ with cterminal GFP or tagRFP fusion tags. We confirmed the predicted localization of GRP within the cell wall with confocal microscopy of $N$. benthamiana leaves transiently expressing a tagRFP-tagged version of the CrGRP (Supplementary Fig. 3). Western blot analyses showed that the protein migrated at the calculated size and does not appear to be secondarily modified in $N$. benthamiana (Fig. 2b). Extracts of these leaves, expressing $C r G R P$, induced ethylene production in a CuRe1-dependent manner like the original $C$. reflexa extract (Fig. 2c). Moreover, when expressing CrGRP in $N$. benthamiana leaves for 5-7 days, clear hypersensitive cell death can be observed only when CuRel is present but not in control plants (Fig. 2d) lacking the receptor. These findings demonstrate that 
CrGRP is the trigger to initiate the CuRe1-dependent defense program.

The minimal peptide epitope of CrGRP. Due to unspecific degradation of the full-length CrGRP during our initial extraction protocol, the extracted forms of the Cuscuta factor were rather small peptides in a range between 2000 and $4000 \mathrm{Da}^{15}$ (Supplementary Table 1). We therefore assumed a minimal peptide motif within the CrGRP full-length protein $(\sim 11.5 \mathrm{kDa})$, which must be sufficient to trigger the defense program. We thus tested a synthetic peptide representing $\mathrm{CrGRP}_{82-106}$ for activity. The peptide was highly active and triggered ethylene production at concentrations $\geq 0.1 \mathrm{nM}$ (Fig. 3a, b), which is in the range we previously estimated for the Cuscuta factor purified from plant extracts ${ }^{15}$. To further narrow down the active motif we tested shortened versions of the peptide, resulting in a 21 aa peptide, termed as crip21 for cysteine-rich peptide 21 , that still retained the full activity found with $\mathrm{CrGRP}_{82-106}$ or full-length CrGRP (Figs. 2a and $3 \mathrm{a}, \mathrm{b}$ ). Peptides further shortened from the $\mathrm{N}$ - or C-terminus showed only reduced activity or no activity, respectively (Fig. 3a and Supplementary Fig. 4). A 15 aa peptide representing the Nterminal sequence present on the purified peptide was inactive (Supplementary Fig. 4), further demonstrating that activity resides in the C-terminal part with the Crip21 motif of CrGRP (Fig. 2a).

Treatment of $C$. reflexa extracts or purified Cuscuta factor with $\mathrm{NH}_{4} \mathrm{OH}$ at $\mathrm{pH} \geq 11$ leads to a total loss of bioactivity and indicated potential secondary modifications on the CrGRP ${ }^{15,16}$. However, synthesized peptides trigger responses in a picomolar range (Fig. 3b and Supplementary Fig. 4) suggesting that no such secondary modifications are required for the bioactivity of Crip21. Much like the purified Cuscuta factor ${ }^{15}$, synthesized Crip21 peptide, comprising no secondary modifications, lost all of its activity when treated with $\mathrm{NH}_{4} \mathrm{OH}$ (Supplementary Fig. 5a). We analyzed the $\mathrm{NH}_{4} \mathrm{OH}$-treated peptide by $\mathrm{MS} / \mathrm{MS}$ and observed multiple reaction products of Crip21 among which the most prominent ones were 82 or 99 Da smaller (Supplementary Fig. 5b), clearly showing that the treatment of Crip21 with $\mathrm{NH}_{4} \mathrm{OH}$ is modifying the peptide itself.

We infiltrated Crip21 into the leaves of resistant S. lycopersicum, susceptible Solanum pennellii and the introgression line IL81-1 lacking $C u R e 1^{15}$, to check whether Crip21 also induces visible HR in tomato. After 7 days, only the cultivated tomato or an introgression line (IL) with functional CuRe1 showed Crip21dependent cell death while S. pennellii and the IL lacking CuRe1 did not (Fig. 3c).

Binding of CrGRP and Crip to the receptor CuRe1. To test for a direct interaction of the peptide epitope Crip with the receptor CuRe1, we n-terminally labelled a 29-aa-long Crip peptide with biotin (bio-Crip29). The bio-Crip29 peptide is an N-terminally $(+4$ aa) and C-terminally ( +4 aa) prolonged Crip21 (Fig. 2a) and was as active as Crip21 (Fig. 3b). The Crip21 minimal epitope has been prolonged to introduce a higher number of Lysine residues to increase the chance for a successful chemical crosslinking of $\mathrm{NH}_{2}$ groups on the ligand with $\mathrm{NH}_{2}$ groups on the receptor. We then examined the interaction of CuRe1 proteins with bio-Crip29 in affinity-crosslinking experiments in planta. N. benthamiana leaves expressing the myc-tagged receptor CuRe1 were first incubated with the bio-Crip29 derivative, either alone or together with an excess of non-modified Crip as competitor, and the leaves were subsequently treated with a chemical cross-linker. When analyzed for the presence of biotin, immunoprecipitates of CuRe1 showed clear labelling which was absent in samples treated with an excess of non-modified Crip as competitor (Fig. 3d). In turn, binding of bioCrip29 was not out-competed when using structurally unrelated peptides such as flg22 (Fig. 3d). These findings demonstrate the CrGRP derived peptide epitope Crip as the specific ligand for the CuRe1 receptor. To corroborate direct protein-protein interaction of the full-length CrGRP with CuRe1 as it may occur under physiological conditions, both proteins were co-expressed with different c-terminal tags and the interaction of both could be demonstrated in co-immunoprecipitation assays (Fig. 3e).

To identify aa-residues of Crip21 which are critical for CuRe1activation, the 21 aa-residues were individually substituted by alanine or serine (substitutions for the cysteines), respectively (Supplementary Table 1). Replacement of the cysteine residues at positions $7,17,20$, and 21 by Serine led to a reduced functionality or in case of $\mathrm{C} 7$ to a complete loss of function. In contrast, the other aa residues seemed less important and had no measurable effects on activity (Supplementary Table 1).

GRP and Crip21 homologs in other plants. When p-blasting the CrGRP or Crip21 aa-sequences against a database of the translated C. campestris genome ${ }^{21}$ and transcriptome ${ }^{22}$, we found a GRP homolog (Supplementary Fig. 6) which also contains a peptide motif (CcCrip21) with a sequence similarity of $\sim 70 \%$ to the C. reflexa Crip21. Especially the glycine residues and the six cysteines are highly conserved (Supplementary Fig. 6). A comparable GRP sequence has been also found in the sequence database of $C$. australis $^{23}$ with the CaCrip21 peptide showing exactly the same 21 aa sequence long peptide as CcCrip21 (Supplementary Fig. 6a). The C. campestris CcCrip21, or $C$. australis CaCrip21, respectively, showed full activity at similar concentrations in CuRe1-dependent ethylene induction (Supplementary Fig. 6b). This corroborates previous findings in which we could show that the defense-triggering Cuscuta factor is also present in other Cuscuta species ${ }^{15}$. In general, GRPs are widely distributed all over the plant kingdom. Even in cultivated tomato (S. lycopersicum) we found a homolog with an aa-sequence similarity of $57 \%$ to CrGRP and we thus assumed the corresponding peptide motif to Crip21, SlCrip21 could serve as an endogenous trigger for tomato CuRe1. We therefore tested the synthesized peptide SlCrip21 in our bioassays where SlCrip21 exhibited only residual activity when applied at concentrations $\geq 1000 \mathrm{nM}$ (Supplementary Figs. 6c and 7).

By substitution of single aa-residues within Crip21 using SlCrip21 as a template (Supplementary Fig. 7), and testing those peptides for bioactivity via CuRe1, we discovered that replacement of the Alanine at position 11 in Crip 21 by a Tyrosine residue (as is the case in SlCrip21) abolished its CuRe1-dependent activity (Supplementary Fig. 7), which is possibly important to avoid autoimmune responses in tomato. However, substituting the tyrosine (Y11) of SlCrip21 by Alanine did not restore activity, indicating that additional changes in the peptide sequence of SlCrip contribute to avoiding self-recognition in tomato (Supplementary Fig. 7). The biological function of the full-length protein SIGRP is unclear and SlGRP may probably play other roles in tomato not related to cellular defense responses and independent of tomato CuRe1. In general, assigned functions of plant GRPs are multifaceted and range from the stabilization of cell walls to hypothesized regulating functions during abiotic and biotic stress reactions ${ }^{24,25}$, which makes it difficult to speculate about the role of the respective GRP in tomato or Cuscuta. Future work will have to reveal what the in vivo function of CrGRP for C. reflexa could be. By BLAST searching for Crip21 peptide homologs, we got hits for this peptide motif related to GRPs of many plant species. Peptides giving the best hits and showing the highest sequence identity to Crip21 were synthesized and tested for their capability to trigger ethylene in samples of CuRe1-expressing $\mathrm{N}$. benthamiana as well as in cultivated tomato (S. lycopersicum; 


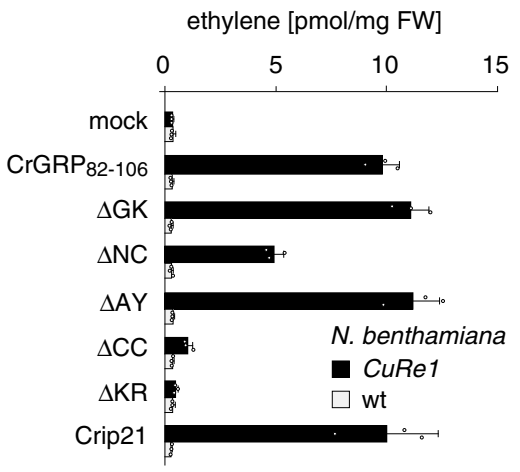

\author{
GKNCGNSGCCGGAYSNGQCKRCCAY \\ NCGNSGCCGGAYSNGQCKRCCAY \\ GNSGCCGGAYSNGQCKRCCAY \\ GKNCGNSGCCGGAYSNGQCKRCC \\ GKNCGNSGCCGGAYSNGQCKR \\ GKNCGNSGCCGGAYSNGQC \\ NCGNSGCCGGAYSNGQCKRCC
}

b

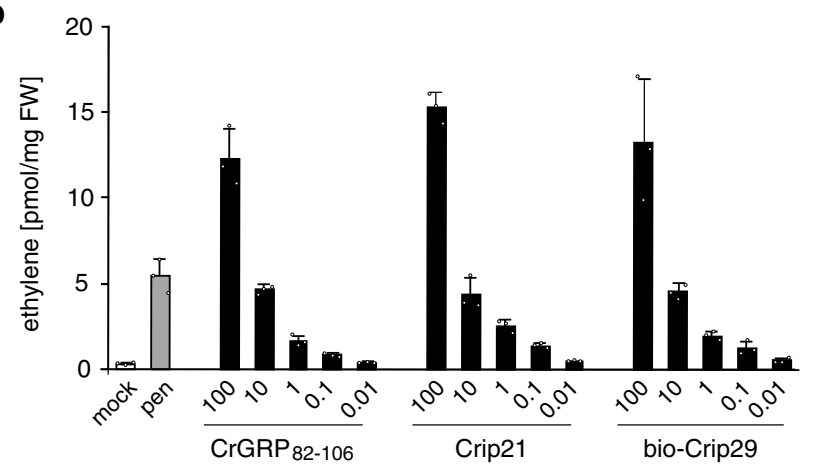

C

native CuRe1

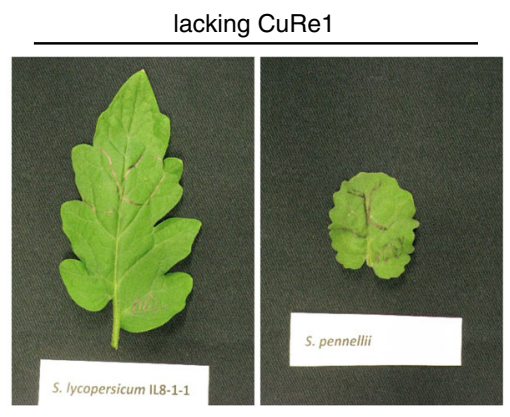

S. Iycopersicum

IL 8-1-1

S. pennellii

d

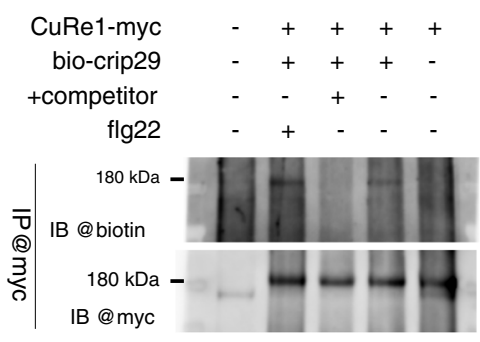

e

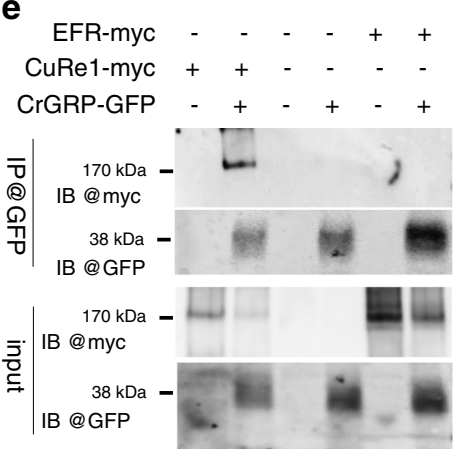

Fig. 3 The peptide epitope crip21 of CrGRP triggers the tomato receptor CuRe1. a Synthesized peptides deriving from CrGRP induce ethylene production in transgenic CuRe1-expressing N. benthamiana. Peptides were applied at concentrations of $1 \mu \mathrm{M}$ each. $\mathbf{b}$ Dose-dependent induction of ethylene by the CrGRP derived, synthesized peptides in CuRe1-expressing N. benthamiana; numbers on $\mathrm{x}$-axis indicate peptide concentrations in $\mathrm{nM}$; bio-crip29 is the $\mathrm{N}$ terminally biotinylated peptide used in binding studies; for a and b: Bovine serum albumin (BSA; $0.01 \mathrm{mg} / \mathrm{ml}$ ) buffered in $25 \mathrm{mM} \mathrm{MES} \mathrm{(pH} \mathrm{5.7)} \mathrm{was} \mathrm{added}$ as mock control; Penicillium extract $(0.05 \mathrm{mg} / \mathrm{ml})$ served as positive control ${ }^{31}$. $\mathrm{FW}$, fresh weight; ethylene measurements show means of three technical replicates; dots indicate single data points; error bars denote SD, representative graphs are shown, experiments were repeated independently more than three times. c crip21 peptide induces HR-type of cell death in a CuRe1-dependent manner. Leaves of S. lycopersicum, an introgression line (IL 12-2) with functional CuRe1and leaves of IL 8-1-1 and S. pennellii (right; both lacking CuRe1) were infiltrated with 100 nM crip21 and photographs were taken 7 days later; painted lines indicate infiltrated leaf area. The effects shown are representative for ten infiltrated leaves per tomato IL or species, respectively; experiments have been independently repeated three times. d Affinity-crosslinking of crip29-biotin with CuRe1 in planta. Solubilized proteins were immune-precipitated and analyzed for myc-tagged (bottom) and biotinylated proteins (top); CrGRP $_{82-106}$ served as competitor; e Co-immunoprecipitation experiments demonstrate interaction of CuRe1 with CrGRP full length protein after co-expression for $\sim 48 \mathrm{~h}$ in planta. 
Supplementary Fig. 6c). Crip21 peptides from blue picotee (Ipomoea nil), Indian lotus (Nelumbo nucifera), and lettuce (Lactuca sativa) were able to induce defense related ethylene production in a CuRe1-dependent manner (Supplementary Fig. 6c). Importantly though, the SlCrip21 peptide from tomato showed only residual activity at $1000 \mathrm{nM}$, suggesting that $\mathrm{CuRe} 1$ could have evolved in Solanum as a perception system for a molecular pattern of non-self that is characteristic for any attacking invader such as dodder described here.

In summary, we showed that CrGRP comprises the molecular pattern with the characteristic Cys-residues that mark the plant parasite C. reflexa as an alien attacker to host plants with the cell surface receptor CuRe1. These findings about the molecular dialogue between host plants and attacking parasitic plants will help to understand resistance during plant-plant interactions and open new possibilities to improve resistance against Cuscuta spp. as well as to design resistance against parasitic plants in general.

\section{Methods}

Cuscuta spp. extract preparation, purification and identification of CrGRP. C. reflexa extract was prepared as described ${ }^{15}$, with modifications outlined below. Cuscuta ssp. shoots were harvested, frozen in liquid nitrogen and freeze-dried for storage. Extraction was performed with $100 \mathrm{mM} \mathrm{HCl}(\mathrm{pH} \sim 1.0)$ at $60^{\circ} \mathrm{C}$ for $16 \mathrm{~h}$. Extract was adjusted to pH 5.5 with $25 \mathrm{mM} \mathrm{MES}$, filtered $(0.22 \mu \mathrm{m}$ MCEM filters, Merck Millipore or for higher volumes through silica gel 60, Macherey-Nagel), loaded on a cation-exchange column (SP Trisacryl ${ }^{\circledR}$ M, Sigma; $25 \mathrm{mM}$ MES $\mathrm{pH}$ 5.5) and eluted with $600 \mathrm{mM} \mathrm{KCl}$. The obtained elution was supplied with $\left(\mathrm{NH}_{4}\right)_{2} \mathrm{SO}_{4}$ to a final salt concentration of $3 \mathrm{M}$; bulk protein was precipitated at $4{ }^{\circ} \mathrm{C}$ and removed while the bioactive protein/peptides stayed in solution. The solution was then desalted by loading on a C18 reversed-phase column (Chromabond, bench-top, $20 \mathrm{mM}$ ammoniumacetate/acetic acid, $\mathrm{pH}$ 4.5) and eluted with $40 \%$ acetonitrile. This pre-purified Cuscuta extract was sequentially separated (Fig. S1) on a strong cation exchange (SCX) column (GE healthcare; Fig. S1A). Active fractions were equilibrated with $25 \mathrm{mM}$ MES (KOH, pH 5.5) and a first run on a C18 reversed phase column (ZORBAX Rx- C18, Agilent) with $20 \mathrm{mM}$ ammonium acetate/acetic acid ( $\mathrm{pH} 4.5)$ and elution with a gradient of acetonitrile $(0-20 \%)$ was performed (Fig. S1B). Active fractions were again pooled and a second run on the same column with $0.1 \%$ acetic acid $(\mathrm{pH} 4.5)$ and elution with a gradient of acetonitrile $(0-20 \%)$. Fractions with highest activity from this second run were further separated on a reversed phase column (Waters, ACQUITY C18 HSST3) equilibrated with $0.1 \%$ formic acid and eluted with a gradient of methanol $(0-30 \%, 60 \mathrm{~min})$. The CuRe1 responsive eluate of this final step was analyzed under similar LC conditions by LC-MS (Waters Acquitiy UPLC - Synapt G2 LC/MS system, electrospray ionization). We correlated distinct masses in these fractions to the CuRe1-dependent responses in transgenic $N$. benthamiana leaf samples. In all, $1 \mu \mathrm{l}$ of the obtained fractions were tested for their capability to induce ethylene production in CuRel transgenic N. benthamiana plants. The identified masses were further analyzed by MS/MS fragmentation studies using an Easy nano-LC (Thermo Scientific) coupled to an LTQ Orbitrap Elite mass specrometer (Thermo Scientific) as previously described ${ }^{26}$ and evaluated with mMass ${ }^{27}$. The aa sequences were calculated manually from the fragment spectra obtained.

The Cuscuta factor $(\mathrm{CuF})$ could be inactivated by incubating it either with $12.5 \% \mathrm{NH}_{4} \mathrm{OH}$ or $70 \%$ ethylamine for $1 \mathrm{~h}$ at $45^{\circ} \mathrm{C}$.

\section{C. reflexa cell wall purification and Pectinase treatment. Lyophilized C. reflexa} was ground to fine powder (liquid nitrogen) and subsequently washed with $70 \%$ Ethanol, Chloroform/Methanol (1:1 v/v), $200 \mathrm{mM} \mathrm{CaCl} 2(5 \mathrm{mM}$ Sodium Acetate pH 4.6), $10 \mathrm{mM}$ EGTA (5 mM Sodium Acetate $\mathrm{pH} 4.6$ ), and $3 \mathrm{M} \mathrm{LiCl}(5 \mathrm{mM}$ Sodium Acetate $\mathrm{pH}$ 4.6). The washed cell wall was dried with Acetone. In all, $\sim 100$ mg purified cell wall was incubated with Pectinase (from Aspergillus niger, SigmaAldrich) or Cellulase (from Trichodoma reesei ATCC 26921, Sigma-Aldrich) according the supplier's guidelines. Extracts, similarly prepared from tomato or tobacco served as controls.

Plant response assays. All obtained extracts or collected fractions after each purification step, as well as the isolated CrGRP or synthesized Crip peptides were tested for bioactivity in the ethylene assay as previously described ${ }^{15}$. For this we cut leaf samples of analyzed plants in $3 \times 3 \mathrm{~mm}$ squares and float them on a water surface. After incubation over night at RT, three leaf pieces are carefully collected into glass tubes $(6 \mathrm{ml})$ with $500 \mu \mathrm{l}$ water. Samples were treated as indicated as well as amounts of used extracts or concentrations of peptides. After treatment tubes were sealed with a rubber plug and incubated at RT on a horizontal shaker (85-100 rpm) for $3 \mathrm{~h}$. All samples were analyzed with a Gaschromatograph (Shimadzu, GC-2014, glass column $3 \mathrm{~mm} \times 1.6 \mathrm{~m}$ with $\mathrm{Al}_{2} \mathrm{O}_{3}$ ) by manually injecting $1 \mathrm{ml}$ of the gaseous phase.
DNA extraction and cloning of CrGRP. C. reflexa plants were grown under long day conditions $(16 \mathrm{~h}$ day $/ 8 \mathrm{~h}$ night $)$ at $22^{\circ} \mathrm{C}$, in a greenhouse. Genomic DNA was extracted from frozen tissue using the Plant DNA Preparation Kit (Jena Biosciences, Germany), and PCR was performed with gene specific primers for the candidate gene (C_ref_r2_000247) CrGRP): FW: ATGAGTTCAAGGGTCTTT CTTCTCC, REV: AGGCTTCGTCGCATCAATGGC; The PCR products were cloned to the $\mathrm{pCR} / \mathrm{GW} / \mathrm{TOPO}$ TA-cloning vector (Invitrogen ${ }^{\mathrm{m}}{ }^{\mathrm{p}}$, Thermo Fisher). Reverse primers without stop codon allowed for C-terminal fusion to a GFP tag after recombining via LR-reaction (LR-clonase ${ }^{\circledast}$ II Plus enzyme mix, Invitrogen ${ }^{\mathrm{m}}$ ) into respective vectors (pB7FWG2.0, pK7FWG2.0, both with C-terminal GFP tag. plant systems biology, university of Gent). For cloning of a $C r G R P$ cDNA construct, total RNA was extracted from tomato plants (RNeasy Plant Mini Kit, Quiagen), and cDNA was synthesized by reverse transcription (First-Strand cDNA Synthesis Kit, GE Healthcare Life Sciences); PCR was performed with primers above. For subcellular localization, CrGRP has been cloned via LR-reaction into a modified version of pGWB660, including a tagRFP 28 .

CrGRP expression and protein isolation. The 35S::CrGRP:GFP construct (in vector pB7FWG2.0; plant systems biology, university of Gent) was transiently transformed into N. benthamiana leaves using Agrobacterium tumefaciens (strain GV3101). A. tumefaciens cultures $\left(\mathrm{OD}_{600}=0.1\right.$ in $10 \mathrm{mM} \mathrm{MgCl}_{2}, 150 \mu \mathrm{M}$ Acetosyringone) were infiltrated into leaves of 4 weeks old $N$. benthamiana plants, according to the described protocol ${ }^{29}$. About $48 \mathrm{~h}$ post infiltration, leaves were harvested, ground under liquid nitrogen to fine powder, supplemented with buffer ( $\sim 3 \mathrm{x}$ volume), and centrifuged ( 45 min, 100,000 rcf, $4^{\circ} \mathrm{C}$ ). The supernatant was then collected for further testing. An extract of $N$. benthamiana leaves expressing GFP alone (pB7WGF2.0) was prepared similarly and served as mock control for treatments.

For monitoring hypersensitive responses (HR) in leaves, $35 \mathrm{~S}: \mathrm{CrGRP}: \mathrm{GFP}$ or 35S::GFP constructs were expressed in either transgenic, CuRe1-expressing or wt $\mathrm{N}$. benthamiana plants. Leaves were infiltrated as described above using the defined volume of $200 \mu \mathrm{l}$; infiltration area was carefully labeled with a black marker. Pictures were taken 7 days post infiltration. For detecting HR-symptoms in tomato plants, two leaves of three plants (six leaves total; per introgression line or wildtype) were infiltrated with $100 \mu \mathrm{l}$ of a $100 \mathrm{nM}$ peptide solution; infiltrated leaf area was labeled with a pen immediately after infiltration and photographs were taken 7 days post peptide infiltration.

Confocal microscopy. Images of transiently transformed $N$. benthamiana were taken 5 days after $A$. tumefaciens infiltration with a Zeiss confocal laser scanning microscope (LSM880, Carl Zeiss Microscopy GmbH, Carl-Zeiss-Promenade 10, 07745 Jena, Germany) and the attached C-Apochromat ×10/0.45 W M27 objective The tag-RFP fluorescence was excited with $561 \mathrm{~nm}$ and emission was detected at $563-607 \mathrm{~nm}$. Autofluorescence of plant cell walls (lignin) was excited at $405 \mathrm{~nm}$ and emission was detected at $410-466 \mathrm{~nm}$. Pinhole, detector gain and digital gain settings were adjusted to provide an optimal balance between fluorescence intensity and background signal. Data were processed with the ZEN 2.3 software.

Binding assays and immunoprecipitation assays. Direct interaction of CrGRP with $\mathrm{CuRe} 1$ was tested by co-immunoprecipitation. For immunoprecipitation, leaves of $N$. benthamiana were transiently transformed with 35S::CuRe1:myc or 35S::CrGRP:GFP alone each, or co-expressed in combination for $\sim 48 \mathrm{~h}$. Leaf material was harvested, frozen in liquid nitrogen and ground to fine powder. Samples of $300 \mathrm{mg}$ were solubilized and used for immunoprecipitation as reported $^{29}$ using $\alpha$-GFP trap Sepharose beads (ChromoTek, IZB Martinsried, Germany) Samples were separated by SDS-PAGE ( $8 \%$ Acrylamide gels) and transferred to nitrocellulose membrane. Western blots were probed using the a-GFP (Acris (now OriGENE) Polyclonal Antibody to GFP; Cat. No.: R1091P; dilution 1:5000 in 5\% BSA; goat; UniProt: P42212) or a-myc (Sigma Polyclonal anti-c-Myc antibody; Cat No.: C3956; dilution: 1:5000 in 5\% BSA; from rabbit; UniProt: P01106) antibodies, diluted according to the instructions of the suppliers, and developed with secondary antibodies conjugated to alkaline phosphatase as described ${ }^{29,30}$ (Sigma; Anti-Goat IgG (whole molecule) - Alkaline Phosphatase antibody produced in rabbit; Cat. No.: A4187, dilution: 1:50,000 in 5\% BSA; OR: Sigma; Anti-Rabbit IgG (whole molecule) - Alkaline Phosphatase antibody produced in goat, Cat. No.: A3687, dilution: 1:50,000 in 5\% BSA).

In vivo cross-linking of biotin-Crip29 (Crip29 aa-sequence: GKGKNCGNSGC CGGAYSNGQCKRCCAYKG) to CuRe1 was performed as described ${ }^{30}$; leaves of $N$. benthamiana expressing 35S::CuRe1:myc, or control plants ( $N$. benthamiana expressing 35S::RLP23:myc) were infiltrated with biotinylated bio-Crip29 (10 nM in $\mathrm{ddH} 2 \mathrm{O}$ ) with or without unlabeled Crip21 (or unlabeled Crip82-106) $(10 \mu \mathrm{M})$ as competitor or with flg22 peptide as competition control. Five minutes after peptide treatment $2 \mathrm{mM}$ EGS (ethylene glycol bis(succinimidyl succinate) in $25 \mathrm{mM}$ HEPES buffer ( $\mathrm{pH} 7.5$ ) was infiltrated into the same leaves for cross-linking of peptides to the receptor proteins. Twenty minutes after cross-linking, leaf samples were harvested and frozen in liquid nitrogen; immunoprecipitations were performed against the myc tag present at the c-terminus of CuRel using myc-trap agarose beads (ChromoTek, IZB Martinsried, Germany) as described above. All peptides, including biotinylated bio-Crip29, were synthesized by GenScript ${ }^{\circledast}$ and ordered with a purity of $>95 \%$. Biotinylated Crip 29 was detected on blots by 
Streptavidine-conjugated Alkaline Phosphatase (Strep-AP, Roche diagnostics; Streptavidin-AP conjugate, Cat. No.: 11089161001, dilution: 1:1000 in 5\% Albumin Fraction V, biotin-free)

Reporting summary. Further information on research design is available in the Nature Research Reporting Summary linked to this article.

\section{Data availability}

Source data are provided with this paper. Any other supporting data are available from the corresponding author upon request.

Received: 20 February 2020; Accepted: 28 September 2020;

Published online: 20 October 2020

References

1. Böhm, H., Albert, I., Fan, L., Reinhard, A. \& Nurnberger, T. Immune receptor complexes at the plant cell surface. Curr. Opin. Plant Biol. 20C, 47-54 (2014).

2. Macho, A. P. \& Zipfel, C. Plant PRRs and the activation of innate immune signaling. Mol. Cell 54, 263-272 (2014).

3. Zipfel, C. \& Oldroyd, G. E. Plant signalling in symbiosis and immunity. Nature 543, 328-336 (2017).

4. Kaiser, B., Vogg, G., Fürst, U. B. \& Albert, M. Parasitic plants of the genus Cuscuta and their interaction with susceptible and resistant host plants. Front. Plant Sci. 6, 45 (2015).

5. Westwood, J. H., Yoder, J. I., Timko, M. P. \& dePamphilis, C. W. The evolution of parasitism in plants. Trends Plant Sci. 15, 227-235 (2010)

6. Spallek, T., Mutuku, M. \& Shirasu, K. The genus Striga: a witch profile. Mol. Plant Pathol. 14, 861-869 (2013).

7. Li, J., Lis, K. E. \& Timko, M. P. Molecular genetics of race-specific resistance of cowpea to Striga gesnerioides (Willd.). Pest Manag. Sci. 65, 520-527 (2009).

8. Li, J. \& Timko, M. P. Gene-for-gene resistance in Striga-cowpea associations. Science 325, 1094 (2009).

9. Duriez, P. et al. A receptor-like kinase enhances sunflower resistance to Orobanche cumana. Nat. Plants 5, 1211-1215 (2019).

10. Yoshida, S., Cui, S., Ichihashi, Y. \& Shirasu, K. The haustorium, a specialized invasive organ in parasitic plants. Annu. Rev. Plant Biol. 67, 643-667 (2016).

11. Dörr, I. Fine structure of intracellular growing cuscuta-hyphae. Protoplasma 67, 123-\& (1969).

12. Dörr, I. Contact of cuscuta-hyphae with sieve tubes of its host plants. Protoplasma 75, 167-\& (1972)

13. Ihl, B., Tutakhil, N., Hagen, A. \& Jacob, F. Studies on cuscuta-reflexa roxb .7. Defense-mechanisms of lycopersicon-esculentum mill. Flora 181, 383-393 (1988).

14. Johnsen, H. R. et al. Cell wall composition profiling of parasitic giant dodder (Cuscuta reflexa) and its hosts: a priori differences and induced changes. New Phytol. 207, 805-816 (2015).

15. Hegenauer, V. et al. Detection of the plant parasite Cuscuta reflexa by a tomato cell surface receptor. Science 353, 478-481 (2016).

16. Hanisch, F. G., Jovanovic, M. \& Peter-Katalinic, J. Glycoprotein identification and localization of O-glycosylation sites by mass spectrometric analysis of deglycosylated/alkylaminylated peptide fragments. Anal. Biochem. 290, 47-59 (2001).

17. Vaughn, K. C. Attachment of the parasitic weed dodder to the host. Protoplasma 219, 227-237 (2002).

18. Vaughn, K. C. Dodder hyphae invade the host: a structural and immunocytochemical characterization. Protoplasma 220, 189-200 (2003).

19. Olsen, S. et al. Getting ready for host invasion: elevated expression and action of xyloglucan endotransglucosylases/hydrolases in developing haustoria of the holoparasitic angiosperm Cuscuta. J. Exp. Bot. 67, 695-708 (2016).

20. Czolpinska, M. \& Rurek, M. Plant glycine-rich proteins in stress response: an emerging, still prospective story. Front. Plant Sci. 9, 302 (2018)

21. Vogel, A. et al. Footprints of parasitism in the genome of the parasitic flowering plant Cuscuta campestris. Nat. Commun. 9, 2515 (2018).

22. Ranjan, A. et al. De novo assembly and characterization of the transcriptome of the parasitic weed Cuscuta pentagona identifies genes associated with plant parasitism. Plant Physiol. 166, 1186-1199 (2014).

23. Sun, G. L. et al. Large-scale gene losses underlie the genome evolution of parasitic plant Cuscuta australis. Nat. Commun. 9, 2683 (2018).

24. Mangeon, A., Junqueira, R. M. \& Sachetto-Martins, G. Functional diversity of the plant glycine-rich proteins superfamily. Plant Signal. Behav. 5, 99-104 (2010).

25. Mangeon, A. et al. The tissue expression pattern of the AtGRP5 regulatory region is controlled by a combination of positive and negative elements. Plant Cell Rep. 29, 461-471 (2010).
26. Franz-Wachtel, M. et al. Global detection of protein kinase D-dependent phosphorylation events in nocodazole-treated human cells. Mol. Cell Proteomics 11, 160-170 (2012).

27. Strohalm, M., Kavan, D., Novak, P., Volny, M. \& Havlicek, V. mMass 3: a cross-platform software environment for precise analysis of mass spectrometric data. Anal. Chem. 82, 4648-4651 (2010).

28. Nakamura, S. et al. Gateway binary vectors with the bialaphos resistance gene, bar, as a selection marker for plant transformation. Biosci. Biotechnol. Biochem. 74, 1315-1319 (2010).

29. Albert, M. et al. A two-hybrid-receptor assay demonstrates heteromer formation as switch-on for plant immune receptors. Plant Physiol. 163, 1504-1509 (2013)

30. Albert, I. et al. An RLP23-SOBIR1-BAK1 complex mediates NLP-triggered immunity. Nat. Plants 1, 15140 (2015).

31. Thuerig, B., Felix, G., Binder, A., Boller, T. \& Tamm, L. An extract of Penicillium chrysogenum elicits early defense-related responses and induces resistance in Arabidopsis thaliana independently of known signalling pathways. Physiol. Mol. Plant Pathol. 67, 180-193 (2005).

\section{Acknowledgements}

The work of M.A. was funded by the German Research Foundation (DFG AL 1426/1-2 and 1-3; AL 1426/4-1). K.K. and J.-A.B. were supported by grant 16-TF-KK from the Tromsø Research Foundation. The work of N.S. was supported by USDA-NIFA (2013 02345). We thank Farid El Kasmi from the ZMBP Tübingen for kindly providing us the modified pGWB660 including the tagRFP. We would further like to thank Rory Pruitt for constructive criticism and critical reading of the manuscript.

\section{Author contributions}

V.H. isolated and identified the C. reflexa GRP as defense trigger. V.H., M.K., B.K., and B.L. pepared Cuscuta extracts and purified the GRP. M.K. did microscope work and photography. J.A.B., A.R., N.S., and K.K. helped with bioinformatics, gave access to unpublished Cuscuta sequencing data and helped with the identification of the GRP gene/RNA. V.H. and P.S. helped with primer design, GRP cloning, expression, and minimal peptide motif identification. I.D.B., J.S., F.L.H.M., B.M., V.H., G.F., and M.S. did mass spec analyses and helped with MS-data interpretation. I.A. and R.B. performed binding studies; B.L. tested peptides for activity in bio-assays. V.H., K.K., T.N., G.F., M.S., and M.A. designed and discussed the experiments. All authors discussed the data referring to their respected experience and helped with interpretations and data analyses. V.H., G.F., K.K., N.S., M.S., and M.A. wrote the manuscript.

\section{Funding}

Open Access funding enabled and organized by Projekt DEAL.

\section{Competing interests}

The authors declare no competing interests.

\section{Additional information}

Supplementary information is available for this paper at https://doi.org/10.1038/s41467 020-19147-4.

Correspondence and requests for materials should be addressed to M.A.

Peer review information Nature Communications thanks the anonymous reviewers for their contribution to the peer review of this work. Peer review reports are available.

Reprints and permission information is available at http://www.nature.com/reprints

Publisher's note Springer Nature remains neutral with regard to jurisdictional claims in published maps and institutional affiliations.

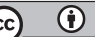

Open Access This article is licensed under a Creative Commons Attribution 4.0 International License, which permits use, sharing, adaptation, distribution and reproduction in any medium or format, as long as you give appropriate credit to the original author(s) and the source, provide a link to the Creative Commons license, and indicate if changes were made. The images or other third party material in this article are included in the article's Creative Commons license, unless indicated otherwise in a credit line to the material. If material is not included in the article's Creative Commons license and your intended use is not permitted by statutory regulation or exceeds the permitted use, you will need to obtain permission directly from the copyright holder. To view a copy of this license, visit http://creativecommons.org/ licenses/by/4.0/.

(C) The Author(s) 2020 